\title{
BMJ Open Theory-based self-management intervention to improve adolescents' asthma control: a cluster randomised controlled trial protocol
}

\author{
Katherine Harris, Gioia Mosler, Jonathan Grigg
}

To cite: Harris K, Mosler G, Grigg J. Theory-based selfmanagement intervention to improve adolescents' asthma control: a cluster randomised controlled trial protocol. BMJ Open 2019;9:e025867. doi:10.1136/ bmjopen-2018-025867

- Prepublication history and additional material for this paper are available online. To view these files, please visit the journal online (http://dx.doi. org/10.1136/bmjopen-2018025867).

Received 6 August 2018 Revised 26 February 2019 Accepted 11 March 2019

\section{Check for updates}

(c) Author(s) (or their employer(s)) 2019. Re-use permitted under CC BY-NC. No commercial re-use. See rights and permissions. Published by BMJ.

Centre for Genomics and Child Health, Queen Mary University of London, London, UK

Correspondence to Dr Katherine Harris; k.harris@qmul.ac.uk

\section{ABSTRACT}

Introduction Asthma-related morbidity and mortality in the UK is higher than elsewhere in Europe. Although the reasons for this are largely unclear, one explanation could be a higher prevalence of poorly controlled asthma in the UK. Findings from our earlier study found that, in a sample of 766 children with asthma, $45.7 \%$ had poorly controlled asthma. Our earlier study also showed that adherence to inhaled corticosteroids was low. Subsequent focus groups identified concerns regarding embarrassment and bullying as barriers to adherence, as well as forgetfulness and incorrect medication beliefs. Following this, a schoolbased self-management intervention has been developed, aimed to improve asthma control and self-management behaviours.

Methods and analysis The theory-based cluster randomised controlled trial tests an intervention comprising two components: (1) a theatre workshop for all children in years 7 and 8, and (2) self-management workshops for children with asthma. The COM-B model was used to guide the development of the intervention. Questionnaire data will be collected in schools at baseline, immediately post intervention, and 3, 6 and 12 months post intervention. The data collected at 6 months will measure the effect of the intervention against the baseline data. The primary outcome will be asthma control, measured using the Asthma Control Test. All the data will be analysed quantitatively using generalised linear and non-linear mixed effects models.

Ethics and dissemination Ethical approval was obtained by the Queen Mary University of London Ethics Committee on 12 April 2018. Regular meetings will be held with key patient and public stakeholders to plan the key messages from this research. Key messages from the study will also be tweeted via the project twitter account (@SchoolsAsthma).The findings of the study will be submitted for presentation at conferences, as well as written into a manuscript.

Trial registration number MGU0400

\section{INTRODUCTION}

\section{Background and rationale}

Approximately 1.1 million children and young people in the UK are living with asthma, making it the most common chronic disease in children in the UK. According to

\section{Strengths and limitations of this study}

This study is a cluster randomised trial, reducing the risk of contamination bias.

- The intervention is guided by a theoretical framework.

- The study includes a strong patient and public involvement focus, and all elements have been tested with children.

- The study will include a volunteer sampling strategy.

- The transferability of the findings to areas outside of London is unclear.

the Global Initiative for Asthma (GINA), ${ }^{1}$ people with good asthma control should not experience troublesome symptoms. Despite this, asthma-related morbidity and mortality in the UK are disproportionally higher than those in most other Western countries; however, the reasons for this remain unclear. In the UK and Ireland, approximately 15\% of the respective populations are living with asthma. ${ }^{2}$ This is in comparison with the rest of Western Europe, where asthma prevalence is approximately 6\%. ${ }^{3}$ In England, asthma mortality among 5-34-year olds is approximately 3.2 per 100000 asthmatics, compared with European nations such as Finland and Sweden, where mortality rates are 1.6 and 2.0 per 100000 asthmatics, respectively. ${ }^{4}$ According to the National Review of Asthma Deaths, ${ }^{5}$ asthma-related deaths in the UK are preventable in up to $65 \%$ of cases. Factors identified in the review that have been found to be associated with asthma-related deaths include poor medication adherence, as well as a poor understanding of the risks related to the condition, especially in children and young people.

Our recent observational study ${ }^{6}$ evaluated current levels of asthma control and self-management in adolescents. The study, in combination with our earlier focus groups, 
informs about current levels of asthma control among adolescents in London and existing barriers to successful self-management. Poor asthma control, poor medication adherence and poor understanding of asthma were identified as unmet needs of secondary school children. We found that $45.7 \%$ of the children in our sample had poor asthma control, as indicated by a score of 19 or less out of 25 on the Asthma Control Test (ACT), and $60.4 \%$ of students did not take their inhaled corticosteroid inhaler as prescribed; $30 \%$ of students did not take their shortacting beta-agonist inhaler when they needed it. The subsequent focus groups highlighted barriers to medication adherence among teenagers. The reported barriers included forgetfulness, incorrect or unhelpful medication beliefs, and social factors, such as discomfort about taking medication at school due to embarrassment and bullying concerns. The focus groups also highlighted low levels of peer awareness and perceived social stigma as a concern among teenagers. Concerns about communication with general practitioners (GPs) were also highlighted. For example, some students expressed concern that they see a different GP each time they have an appointment, and each GP says something different. Moreover, some students felt that it was difficult to understand the medical terminology often used in consultations, which made it difficult to follow doctor recommendations.

A recent systematic review of school-based self-management interventions, conducted by $\mathrm{KH}$ and JG with colleagues at the Institute of Education and Cochrane, ${ }^{7}$ conducted a process evaluation ${ }^{8}$ and meta-analyses on included studies looking at school-based self-management interventions for children with asthma. The outcomes from this review identified that school-based self-management interventions are successful at improving children's outcomes across several areas, including reduced rates of unscheduled care, improved health-related quality of life, and improved medication use. The process evaluation component of the systematic review furthermore identified that a theoretical framework is an important component of intervention implementation success.

Improving asthma understanding, self-efficacy, and unhelpful beliefs towards asthma and its treatment are key to improving adherence and self-management. ${ }^{9}$ Better understanding and appropriate beliefs can empower teenagers to take control of their asthma, particularly when preparing for the transition to adult care. The effects of poor self-management and non-adherence can also last into adulthood, if a lack of self-management skills and awareness remains.

Following the findings above, and the Medical Research Council (MRC) framework for developing complex interventions, ${ }^{10}$ a preliminary theory-based multifaceted intervention has been developed. This draws on our earlier work $^{67}$ and theory. The intervention aims to improve asthma self-management and subsequent asthma control in young people. The protocol for this study has been developed in accordance with the SPIRIT guidelines, found in the online supplementary file.

\section{Intervention development}

The development of the intervention is theory driven and addresses barriers to successful self-management. The PRECEDE-PROCEED model was used to conduct the initial diagnosis of social, epidemiological, educational and administrative diagnosis. We then used the behaviour change wheel ${ }^{11}$ as a framework to translate identified behaviours into specific interventions with specific translation into behaviour change techniques to maximise the transparency of the intervention and understanding of the processes of action.

The intervention has been developed according to the MRC guidelines for the development of complex intervention. ${ }^{10}$ The intervention furthermore followed the steps of the PRECEDE-PROCEED model for health promotion, which emphasises a diagnostic phase, before implementation and evaluation of an intervention. For its diagnosis, the intervention was based on evidence from a systematic literature review, as well as from questionnaire and focus group data from a previous study. The main behaviour was identified as improved self-management, which was divided in six sub-behaviours, including adherence to medication, trigger avoidance and empowerment to self-manage. Barriers to each sub-behaviour were then mapped according to the COM-B framework by Michie et al, ${ }^{11}$ which identifies capabilities, opportunities and motivation of the (sub-) behaviour.

These barriers to improved self-management of asthma were translated into behavioural targets that we wanted to set as part of the intervention, such as 'awareness of situations in which they could forget their medication', 'understanding about different triggers and how to mitigate their effect' or 'understanding of limitations and possibilities of a life with asthma'. As a next step, each of the behavioural targets fed into the development of intervention elements. The development of each element started with an idea-finding phase, in close collaboration with learning experts, followed by reviews from behavioural and medical experts, as well as input from the target audience. This idea-finding and review process was repeated until an engaging and effective solution for the intervention was found. The elements of the intervention were subsequently analysed using the Behavioural Change Taxonomy from Michie et al. ${ }^{12}$

\section{Research question}

The primary research question is, what is the effect of a school-based self-management intervention on health outcomes for children with asthma, compared with usual care?

The secondary research question is comparing the effect of two different school-based interventions on outcomes for children with asthma: is increasing peer awareness alone sufficient to improve health outcomes for children with asthma? 


\section{Study objectives}

The primary objective of this study is to test the effectiveness of an intervention to improve asthma control in adolescents with asthma. The secondary objective is to raise awareness of asthma in schools among peers. This will be addressed through the delivery of a theatre workshop, in collaboration with Greenwich and Lewisham Young People's Theatre (GLYPT) Company.

\section{Study design}

This study is a cluster randomised trial, with schools acting as the unit of allocation.

\section{METHODS AND ANALYSIS}

\section{Study setting and recruitment strategy}

All schools in London will be invited to take part. The schools will be contacted via email, letter and phone call, and invited to participate. Schools could choose to participate by responding to the letter or email. This recruitment strategy has been selected as it has been used in our previous school-based research and has achieved an appropriately diverse sample. ${ }^{6}$ To maintain anonymity, the names of the schools will be removed from all publications and replaced with a number. Each component of the intervention has been tested and reviewed by children and young people with asthma.

\section{Eligibility criteria}

The inclusion criteria states that children with asthma will be eligible to participate if they have doctor-diagnosed asthma, are in year 7 or eight at secondary school (aged 11-14 years) and are attending the school in which the intervention is delivered. For the theatre component of the intervention, all children in years 7 and 8 will be eligible to participate. No inclusion criteria will be placed on schools. Opt-out consent for the intervention will be obtained from all parents of children with asthma, followed by student assent, which will be collected from the students on the morning of the intervention. The parents have 2 weeks prior to the intervention to opt out, if they do not wish for their child to participate. A copy of the parent information sheet and withdrawal form can be found in the online supplementary file. For the children without asthma, consent to participate in the theatre workshop will be provided by the school. This consent procedure was considered most appropriate, as it was used in our earlier school-based research, and was in accordance with preferences from teachers.

\section{Intervention}

The intervention will include three study arms, and schools will be randomly allocated to one of the arms:

\section{Asthma workshop and theatre group}

The asthma workshop and theatre group will receive the self-management workshops for asthmatic children, and a theatre performance for the whole year group.

\section{Theatre-only group}

The theatre-only group will receive the theatre performance only, for the whole year group, without the added self-management workshops for children with asthma. This arm is included to identify whether raising awareness among peers alone is sufficient to change self-management behaviours among children with asthma.

\section{Control group}

The control groups will receive usual care for the duration of the intervention, to measure the effectiveness of the intervention against usual care. Children in the control schools will receive the full intervention at the end of the study, when all the data have been collected.

The intervention workshops will be delivered by the learning and outreach team, under the supervision of the postdoctoral researcher. GLYPT will deliver the theatre performance in schools. The learning and outreach team will remain unblinded to the school's allocation group. The postdoctoral researcher will remain blinded to the treatment group during the analysis. Any data that could reveal the allocation group during analysis (eg, date of data collection) will be removed by the learning and outreach team prior to analysis. All identifiable data that are collected during the trial will be removed for analysis and will be replaced with a number.

\section{Intervention implementation}

The intervention will be delivered in two parts. First, the theatre workshops will be delivered to all students in years 7 and 8 in secondary schools to facilitate awareness and understanding of asthma among the direct peer group. At the end of the theatre performance, the main character will stay in role and will encourage audience participation and discussion on the play. Our earlier focus groups identified barriers to medication adherence among teenagers in schools, including a belief that their peers do not understand asthma or medication. Through changing attitudes and awareness among peers at school, students with asthma should feel less concerned about the social barriers that currently prevent positive self-management behaviours.

Second, the four self-management workshops will be delivered only to children with asthma, within 2 weeks of delivering the theatre workshop. Each self-management workshop will last approximately 1 hour and will take place in the intervention schools over the course of one school day, to reduce the risk of attrition within the workshops. The workshops will include a series of games, role-play, media (films), and discussions. The main topics will include general knowledge and understanding of asthma, GP communication, asthma triggers and symptoms, medication and emergency response, and self-management techniques and goal setting. Schools will also receive a toolkit, which will include emergency response posters and advice on asthma-friendly schools.

At the end of the workshops, the children will be given a toolkit, including an asthma action plan, information 
on where to find out more about asthma and the details of a smartphone app about asthma. The control schools will receive the intervention at the end of the study, once all of the follow-up data have been collected. The data will be collected using questionnaires and will be completed using a tablet device. The online questionnaires will ensure that all questions are answered before a child can move on to the next question, to reduce the risk of missing data. A copy of the online questionnaire that will be used can be found in the online supplementary file.

The control schools will complete the online questionnaires at baseline, approximately 2 weeks later (in line with the completion of workshops), and then 3, 6 and 12 months post intervention. The theatre-only schools and the full-intervention schools will complete the questionnaires at baseline, immediately after the theatre performance, immediately after the workshops, and 3, 6 and 12 months post intervention. The children will be informed of their right to withdraw at any time from the study, without consequence, and their parents will be informed of the study and will be able to withdraw their child from the study before it begins. As this is a pilot study, a full evaluation of the trial will be conducted before a decision is made to deliver the intervention as a full-scale study.

The target population for this study is children with asthma in years 7 and 8 , who are attending secondary school in London. For the theatre performance, the whole-year group (both children with and without asthma) will be invited to participate. Participation will be offered to all secondary schools in London. The schools will be recruited via established recruitment strategies. This includes targeted emails and phone calls to dedicated teachers at each school, to inform them of the research. The schools will be responsible for identifying eligible children (both asthmatic and non-asthmatic) and disseminating the information sheets and withdrawal forms to parents. A copy of the information sheet to parents is found in the online supplementary file.

\section{Patient and Public Involvement}

The development of the intervention was informed by earlier focus groups, conducted with 56 children and young people with asthma across four London secondary schools. During these focus groups, the potential for a school-based intervention was discussed, including the topics that should be included. Findings from our earlier questionnaire ${ }^{6}$ were also presented at a lay research advisory panel, consisting of approximately 12 adults, some of whom had asthma themselves or cared for someone with asthma. During this panel meeting, the findings of the questionnaire and the development of the intervention were discussed, including plans for engagement with children and teenagers for the duration of the intervention.

Each individual component of the intervention has been tested with children and young people in schools and hospital clinics. A pilot study of the full workshops has also been conducted in three London secondary schools, with approximately 60 children and young people with asthma. Following this testing, the wording of the questionnaire has been tweaked to ensure that it is easily understood by the target cohort, and this change was approved by the research ethics committee. The format of the workshops has also been amended. The theatre performance has also been tested in nine schools with 1814 children and young people, both with and without asthma. A documentary about the theatre performance has been produced, including feedback from children and young people and teachers.

\section{Consent}

The parents of eligible children will be sent a study information sheet and withdrawal form by the school. The parents will be given 2 weeks to opt their child out, if they do not wish for their child to participate. The children will also provide written assent on the morning of the study to confirm they are happy to take part. This will be collected by the research team and will be signed by the postdoctoral researcher for audit purposes.

\section{Study outcomes and time points}

The primary outcome in this study is asthma control. The secondary outcomes are medication adherence, unscheduled care and school absences, asthma attitudes, asthma knowledge and beliefs about asthma medication. Baseline data will be collected, followed by immediately post theatre, post workshops, and 3, 6 and 12 months post intervention. The data collected at 6 months will be used for comparison with the baseline data to test the effect of the intervention. The data collected at 12 months will be used to measure whether any changes in behaviour are seen are permanent.

\section{Sample size}

Adjusting for a $15 \%$ attrition rate, a minimum of 360 children with asthma are required for this study. According to the power calculation, at least 18 schools will be recruited (six schools in each arm of the intervention, 20 children with asthma from each school). This attrition rate was based on previous school-based research, which used a $10 \%$ attrition rate after adjustments. Following this, we included a $15 \%$ attrition rate to allow for a more conservative estimate. Maintaining allocation concealment, the schools will be randomised to one of the three intervention arms. This sample size was calculated based on a power calculation, using ACT scores as the primary outcome measure. This sample size has tested for a threepoint difference in ACT score between intervention and control groups, with an SD of 4.5, which is taken from our previous school-based work, ${ }^{13}$ which gives an effect size of 0.67 . The error rate is assumed to be $0 \%$ as the students will input the data directly on iPads, which are checked after for completion, and a $15 \%$ attrition rate has been included. A type II error rate of $20 \%$ and a type I error rate of $5 \%$ were chosen. This is a cluster randomised trial, and an intraclass correlation coefficient (ICC) of 0.05 
will be used. This power analysis was calculated using $\mathrm{R}$ language, specifically the 'pwr' package. ${ }^{1415}$

\section{Randomisation}

The schools will be randomly allocated to one of three arms of the intervention using an online randomisation tool (found here: https://www.randomlists.com/teamgenerator). The schools will be unaware of which treatment arm they have been allocated to. All school visits will be scheduled via email. As the control schools will receive the full intervention at the end, all schools will be aware that they will receive the intervention at some point during the study period. The postdoctoral researcher will lead the study, under the supervision of the principal investigator (PI), and will be responsible for generating the allocation sequence and for assigning schools to the treatment arm.

\section{Data collection}

The data will be collected using an online questionnaire, including a series of validated measures. The primary outcome (asthma control) will be measured using the validated ACT. ${ }^{16}$ The ACT is valid in children aged 12 years and older, with a childhood version for younger children. An ACT score of 19 or below reflects the cut-off score with the best sensitivity and specificity for predicting poor asthma control. ${ }^{16}$ A recent European Respiratory Society Taskforce also recommends more clinical monitoring in children who score 19 or less. ${ }^{17}$

The secondary outcomes will be measured using several measures. Adherence to medication will be assessed using the Medication Adherence Rating Scale (MARS). ${ }^{18}$ This validated scale has been tested in adults with asthma and has shown good reliability and validity and psychometric performance among a low-income population. ${ }^{19}$ The MARS tool has also been validated in other conditions, such as psychosis. ${ }^{18}$ Illness perceptions will be measured using the Brief Illness Perception Questionnaire. ${ }^{20}$ This tool has been widely used across a range of conditions, with a range of different population groups, and has shown good concurrent validity. ${ }^{21}$ Beliefs about medicines will be measured using the Beliefs about Medicine Questionnaire, ${ }^{22}$ which has been shown to accurately assess medication beliefs among patients with a range of chronic conditions, including asthma. ${ }^{22}$ Asthma knowledge will be measured using a scale developed by the research team and tested among children with asthma in clinics, as no validated scale currently exists. Unscheduled care and school absences will also be measured using the scales from our earlier study. ${ }^{13}$

\section{Data management}

All questionnaire data will be collected using an online questionnaire and stored in Excel and SPSS version 25 using a password-protected file. This will be managed by the postdoctoral researcher at the university only. Access to data files will be provided on an 'as-needs' basis. All data will be uploaded directly from the online questionnaire site direct to Microsoft Excel. All paper versions of the questionnaire will be entered manually into an excel database by the postdoctoral researcher. A copy of the questionnaire can be found in the online supplementary file.

\section{Statistical methods}

No interim analyses will be conducted; however, the data will be cleaned as they are collected. The full analysis will first be conducted, using an intention-to-treat model; we will obtain crude estimates, adjusted to account for two covariates (gender and ethnicity). For any skewed continuous data, the maximum, upper quartile, median, lower quartile and minimum data will be presented. If the continuous data are normally distributed, the mean, $\mathrm{SD}$, median, minimum, maximum and number of observations will be reported. All categorical data will be summarised in terms of frequency counts and percentages. An example of tables that will be used to present the data can be found in the online supplementary file.

\section{Primary outcome}

The primary outcome (asthma control) will be compared between the full intervention group and the control group, using a mixed-effect model. This will be largely measured through change in ACT scores; however, the binary outcome for this variable (poor vs good control) will be used in some subgroup analyses. A cut-off score of 19 will be used; scores of 19 or less will be considered indicative of poor asthma control. The size of the difference between the full intervention group and the control group will be expressed as an OR, including a 95\% CI along with $\mathrm{p}$ values. This will be taken from a logistic regression mixed-effect model and a linear mixed-effect model for ACT score. The mixed-effect model was adjusted for the ICC in the power calculation, using an ICC from a similar questionnaire outcome for asthma. The mixed-effect model will used a random intercept model for clusters (schools). A random gradient model will also be conducted as a sensitivity analysis. Children will be individuals within that cluster; their data will be recorded at follow-up and compared with their baseline as a covariate. All the children in the sample will have a shared error distribution and the ICC of 0.05 from the study will be recorded.

\section{Secondary outcomes}

For medication adherence, beliefs about medicines and illness perceptions, a generalised linear mixed-effect model will be conducted. For any count data, this will be a Poisson model; a logistic model will be used for all binary data, controlling for socioeconomic status, using the school postcode as a predictor. For healthcare uses, school attendance and asthma knowledge, a generalised non-linear mixed-effect model will be conducted. For the knowledge about asthma variable, the 'do not know' responses will be considered incorrect, to enable this variable to be considered as a binary outcome. 


\section{Additional analyses}

To compare whether the theatre performance alone is sufficient to change behaviour among children with asthma, a non-inferiority trial will be conducted, using a non-inferiority CI framework, to compare the differences in outcomes between the full intervention group and the theatre-only group, using intention-to-treat analyses. The estimated marginal difference in this non-inferiority trial will be three points on the ACT score, as this is the minimal clinical important difference.

\section{Data monitoring and safety}

As this is a pilot study, no official data monitoring committee will be in place. However, all intervention elements will be carried out in accordance with the protocol and the approval given by the research ethics committee. A risk assessment will also be given to each school.

Any children who report any adverse effects from their asthma (eg, frequent asthma attacks) or who report any other reasons for concern will be reported to the school and the PI of the study. All children will be given information resources, such as Asthma UK, and will be advised to contact their GP if they have any questions or concerns about their asthma or asthma medication.

\section{ETHICS AND DISSEMINATION}

Regular meetings will be held with key patient and public involvement stakeholder groups to plan key messages from our research. This will include the lay research advisory panel at the National Institute of Health Research (NIHR) Collaborations for Leadership in Applied Health Research and Care North Thames, as well as teachers, teenagers and parents. Established channels of social media will be used to disseminate the findings of the intervention. This will include the established Twitter account for the project (@SchoolsAsthma) to reach key organisations and tweet important messages related to this work. The team will also work closely with established partners, such as the Healthy London Partnership, AUKCAR and the Asthma UK knowledge exchange team to disseminate the findings to the media and relevant stakeholders, and to continue developing this research.

The participating schools will receive descriptive statistics for their school only, and will be informed of any publications that arise from this trial. The findings of the intervention will be submitted for presentation at national and international conferences. We will also seek to present our findings at local authority health and wellbeing boards. The findings from this study will be written into a manuscript and will be submitted to a peer-reviewed journal. If successful, a grant application will also be submitted to the NIHR to support the implementation of the intervention across the UK. The project research approach will also be documented through a short documentary film, which will be available on the research study website.
The protocol for this study has been registered, and the full dataset will be made available (with identifiable information removed) for the purposes of publication.

Acknowledgements The authors thank the National Institute of Health Research Collaborations for Leadership in Applied Health Research and Care North Thames for their continued support of this project, particularly of the development of the individual components for this intervention, and Dr Chris Newby for his support in the analysis.

Contributors JG is the principal investigator for the study, and planned and provided the overall supervision to the rest of the team. KH contributed to the planning of the study with GM and will be responsible for the data collection and analysis, as well as for disseminating the findings at conferences and in peerreviewed journals. KH also obtained ethical approval and prepared the manuscript of the protocol. GM contributed to the planning of the study and was responsible for the development of the intervention components, alongside KH. GM also manages the Learning and Outreach team, who also worked on the development of the intervention, and will be responsible for the school liaison and recruitment.

Funding This work was supported by Bart's Charity, grant reference MGU0400. This research was supported by the National Institute for Health Research (NIHR) Collaboration for Leadership in Applied Health Research and Care North Thames at Bart's Health National Health Service (NHS) Trust. The views expressed in this article are those of the authors and not necessarily those of the NHS, the NIHR or the Department of Health and Social Care.

Competing interests None declared.

Patient consent for publication Not required.

Ethics approval Ethical approval for this study was obtained from Queen Mary University of London Research Ethics Committee on 12 April 2018 (REC reference number: QMERC2017/77).

Provenance and peer review Not commissioned; externally peer reviewed.

Open access This is an open access article distributed in accordance with the Creative Commons Attribution Non Commercial (CC BY-NC 4.0) license, which permits others to distribute, remix, adapt, build upon this work non-commercially, and license their derivative works on different terms, provided the original work is properly cited, appropriate credit is given, any changes made indicated, and the use is non-commercial. See: http://creativecommons.org/licenses/by-nc/4.0/.

\section{REFERENCES}

1. (GINA) GIfA. Global strategy for asthma management and prevention. 2016.

2. Braman SS. The global burden of Asthma. Chest 2006;130:4S-12.

3. GlfA. From the global strategy for asthma management and prevention. GIfA, 2015.

4. Masoli M, Fabian D, Holt S, et al. The global burden of asthma: executive summary of the GINA Dissemination Committee report. Allergy 2004;59:469-78.

5. Levy M, Andrews R, Buckingham R, et al. Why asthma still kills: the national review of asthma deaths (NRAD): Royal College of Physcians, 2014.

6. Harris K, Mosler G, Williams SA, et al. Asthma control in London secondary school children. J Asthma 2017;54:1-8.

7. Harris K, Kneale D, Lasserson TJ, et al. School-based selfmanagement interventions for asthma in children and adolescents: a mixed methods systematic review. Cochrane Database Syst Rev 2019;1:CD011651.

8. Thomas J, O'Mara-Eves A, Brunton G. Using qualitative comparative analysis (QCA) in systematic reviews of complex interventions: a worked example. Syst Rev 2014;3:1.

9. Horne R. Compliance, adherence, and concordance: implications for asthma treatment. Chest 2006;130:65S-72.

10. Campbell M, Fitzpatrick $R$, Haines $A$, et al. Framework for design and evaluation of complex interventions to improve health. BMJ 2000;321:694-696.

11. Michie S, van Stralen MM, West R. The behaviour change wheel: a new method for characterising and designing behaviour change interventions. Implement Sci 2011;6:42.

12. Michie S, Richardson M, Johnston $M$, et al. The behavior change technique taxonomy (v1) of 93 hierarchically clustered techniques: building an international consensus for the reporting of behavior change interventions. Ann Behav Med 2013;46:81-95. 
13. Harris $\mathrm{K}$, Mosler G, Williams SA, et al. Asthma control in London secondary school children. J Asthma 2017;54:1033-40.

14. Team RC. R Foundation for statistical computing. Vienna, Austria: Team RC, 2014.

15. Champely S, Ekstrom C, Dalgaard P, et al. Package 'pwr'. 2018.

16. Schatz M, Sorkness CA, Li JT, et al. Asthma Control Test: reliability, validity, and responsiveness in patients not previously followed by asthma specialists. J Allergy Clin Immunol 2006;117:549-56.

17. Brand PL, Mäkelä MJ, Szefler SJ, et al. Monitoring asthma in childhood: symptoms, exacerbations and quality of life. Eur Respir Rev 2015;24:ERJ-00888-02014.

18. Thompson K, Kulkarni J, Sergejew AA. Reliability and validity of a new medication adherence rating scale (mars) for the psychoses. Schizophr Res 2000;42:241-7.
19. Cohen JL, Mann DM, Wisnivesky JP, et al. Assessing the validity of self-reported medication adherence among inner-city asthmatic adults: the Medication Adherence Report Scale for Asthma. Ann Allergy Asthma Immunol 2009;103:325-31.

20. Broadbent E, Petrie KJ, Main J, et al. The brief illness perception questionnaire. J Psychosom Res 2006;60:631-7.

21. Broadbent $\mathrm{E}$, Wilkes $\mathrm{C}$, Koschwanez $\mathrm{H}$, et al. A systematic review and meta-analysis of the Brief Illness Perception Questionnaire. Psychol Health 2015;30:1361-85.

22. Horne R, Weinman J, Hankins M. The beliefs about medicines questionnaire: the development and evaluation of a new method for assessing the cognitive representation of medication. Psychol Health 1999;14:1-24. 